\title{
DIMENSION GROUPS ASSOCIATED TO $\beta$-EXPANSIONS
}

\author{
TERESA BATES, TOKE MEIER CARLSEN and SØREN EILERS*
}

(Dedicated to the memory of Gert K. Pedersen)

\begin{abstract}
Completing work by Shultz on one hand and by Katayama, Matsumoto, and Watatani on the other, we prove that a priori different dimension groups associated to $\beta$-expansions in fact coincide.
\end{abstract}

\section{Introduction}

Two different constructions associate dimension groups to $\beta$-expansions. Here, a dimension group ([9], [10]) is an ordered abelian group which is unperforated and has the Riesz properties, and $\beta$-expansions ([18], [17], [1], [19]) are elements of $\{0, \ldots,\lceil\beta\rceil-1\}^{\mathrm{N}_{0}}$ of the form

$$
\left(b_{n}\right)_{n \in \mathrm{N}_{0}}=\left(\left\lfloor\beta T_{\beta}^{n}(x)\right\rfloor\right)_{n \in \mathrm{N}_{0}}
$$

with $x \in[0,1)$, where $\beta>1$ is fixed and $T_{\beta}$ is the $\beta$-transformation

$$
T_{\beta}:[0,1) \rightarrow[0,1) \quad T_{\beta}(x)=\beta x-\lfloor\beta x\rfloor .
$$

The terminology is motivated by the observation

$$
x=\sum_{n=0}^{\infty} b_{n} \beta^{-n-1}
$$

and we shall work mainly with the closure of the set of all such $\beta$-expansions which is denoted as the $\beta$-shift $X_{\beta}$, thinking of this as a symbolic representation of orbits under $T_{\beta}$ as indicated in Figure 1.

The first such construction, considered in [12], involves the fixed point algebra $\mathscr{F}_{\beta}^{\infty}$ for the so-called gauge action of the $C^{*}$-algebras associated by Matsumoto to any shift space ([14]). As noted in [5, Corollary 3.3], in this case the two different ways to build such $C^{*}$-algebras coincide, but the reader

\footnotetext{
* This research has been supported by the EU-Network Quantum Spaces \& Noncommutative Geometry (HPRN-CT-2002-00280).

Received March 28, 2006.
} 


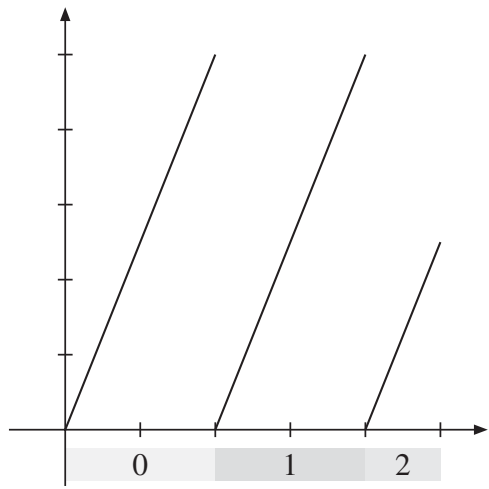

FiguRE 1. $\beta=5 / 2$

may still find the alternative presentations in [3] and [6] useful. This fixed point algebra is an approximately finite (or " $A F$ ", cf. [2]) $C^{*}$-algebra, and its ordered $K_{0}$-group is a dimension group.

The second construction, introduced in [20] and studied further in [21], is a special case of a direct construction valid for any interval map, specialized to the map $T_{\beta}$ defined above.

Since dimension groups with distinguished order units and unital $A F$ algebras are in 1-1 correspondence ([9], [8]) it is just a matter of perspective whether or not one considers the dimension groups or $A F$ algebras, as made clear in [15] and [7]. We shall choose the former as it harmonizes with our proof.

Setting off from the work of Katayama et al we shall work with the ordered $K_{0}$-group of the fixed point algebra $\mathscr{F}_{\beta}^{\infty}$ and denote it by $\left(\Delta_{\beta}, \Delta_{\beta}^{+}\right)$where $\Delta_{\beta}^{+}$ denotes the cone of positive elements in $\Delta_{\beta}$. The importance of this object (cf. [15], [16]) stems from the fact that it is a conjugacy invariant for the underlying shift space, and that it generalizes the dimension groups used in the classification of shifts of finite type up to shift equivalence.

In fact, we shall work with a triple comprising the dimension group, the automorphism $\tau_{\beta}$ acting on it, and a distinguished order unit for the dimension group. The collection $\left(\Delta_{\beta}, \Delta_{\beta}^{+}, \tau_{\beta}\right)$ is referred to as the dimension triple of $X_{\beta}$ (cf. [13] and [16]; notice however that in [16] the automorphism $\tau_{\beta}^{-1}$ is used in place of $\tau_{\beta}$ ).

By [21, Proposition 10.6] the two constructions give the same dimension groups when the $\beta$-shift is sofic (cf. [13]). This is seen by comparing explicit computations of the dimension groups in [21, Proposition 10.3] and [12, Theorem 6.1]. But since the dimension group of the fixed point algebras of the $C^{*}$-algebras associated by Matsumoto to the $\beta$-shift $X_{\beta}$ is only determined 
as a group in [12], the problem of determining whether the dimension groups coincide in general has been left open.

It is the purpose of this note to compute the ordered dimension group in the non-sofic case, thus proving that the two dimension groups coincide in this case as well. This is achieved by a direct computation in combination with a result of Ito and Takahashi ([11]) proving, roughly speaking, that a certain sequence of numbers $\# \mathscr{P}_{n}(x)$ grow as $\beta^{n}$ uniformly in $x$. Apart from importing that result, we are thus able to provide a self-contained proof.

We need to point out a minor inconsistency in [12] of relevance to our work. In the proof of Case 1 of [12, Theorem 1] it is stated, correctly as proved in [12, Corollary 4.6], that the dimension group in the non-sofic case is always isomorphic to a sum of infinitely many copies of Z. However, in the statement of [12, Theorem 1] it is claimed that the dimension group is isomorphic, as a group, to

$$
\mathrm{Z}\left[\beta, \beta^{-1}\right]
$$

and while this is true for most $\beta$, it fails for instance for rational $\beta$ such as $\beta=3 / 2$ where all elements are divisible by 6 .

\section{Preliminaries}

The real numbers will be denoted by $R$, the integers by $Z$, the positive integers by $\mathrm{N}$ and the non-negative integers by $\mathrm{N}_{0}$. We will denote integers by $i, k, l, m, n$ and $b$.

The $\beta$-shift space introduced above (the reader is directed to [12] or [11] for a more detailed definition) will be denoted by $X_{\beta}$, and $\sigma$ will be the shift mapping on $X_{\beta}$. The alphabet $\{0,1, \ldots,\lceil\beta\rceil-1\}$ of $X_{\beta}$ will be denoted by $a$, and we will denote a letter of $a$ by $a$. We will denote the lexicographic order on both $n$-tuples $\mathfrak{a}^{n}$, infinite sequences $\mathfrak{a}^{\mathrm{N}_{0}}$ and finite words of arbitrary length $\mathfrak{a}^{*}$ by $\geq$. The set $X_{\beta}$ has a maximal element under this order (cf. e.g. [12] or [1]) which we shall denote by $\zeta$. Other elements of $X_{\beta}$ will be denoted by $\eta, \xi$ and $\varepsilon$. If $\eta \in X_{\beta}$ and $n \in \mathbf{N}_{0}$, then we will by $\eta_{n}$ denote the $n+1$ st letter of $\eta$. We denote the language of $X_{\beta}$ by $W$, elements of $W$ by $u$ and the length of these elements by $|u|$. If $k \in\{1,2, \ldots,|u|\}$, then $u_{k}$ will denote the $k$ th letter of $u$. Let for $n \in \mathrm{N}_{0}$ the set $W_{n}$ be given by

$$
W_{n}=\{u \in W|| u \mid=n\} .
$$

We then have (cf. [1, p. 136]) that the following identity holds:

$$
W_{n}=\left\{u_{1} u_{2} \cdots u_{n} \in \mathfrak{a}^{n} \mid u_{k} u_{k+1} \cdots u_{n} \leq \zeta_{1} \zeta_{2} \cdots \zeta_{n+1-k} \text { for all } 1 \leq k \leq n\right\} .
$$


For a set $A$, we will by $1_{A}$ denote the characteristic function of $A$. We write, for $\eta \in X_{\beta}$,

$$
\mathscr{P}_{n}(\eta)=\left\{u \in W_{n} \mid u \eta \in X_{\beta}\right\}
$$

and call this set the set of pasts of length $n$ for $\eta$. Equality of $n$-pasts induces the $n$-past equivalence relation which will be denoted by $\sim_{n}$, and $[\eta]_{n}$ will be the $n$-past equivalence class of $\eta$. We will by $\Omega_{X_{\beta}}$ denote the space

$$
\left\{\left(\left[x_{n}\right]_{n}\right)_{n \in \mathrm{N}_{0}} \in \prod_{n \in \mathrm{N}_{0}} X_{\beta} / \sim_{n} \mid \forall n \in \mathrm{N}_{0}: x_{n} \sim_{n} x_{n+1}\right\}
$$

which is a projective limit of the set of $n$-past equivalence classes, cf. [4, 2.5]. Elements of $\Omega_{X_{\beta}}$ will be denoted by $x, y$ and $\left(\left[x_{n}\right]_{n}\right)_{n \in \mathrm{N}_{0}}$. So $x_{n}$ will always denote an element of $X_{\beta}$ and not an element of $a$. We define an order $\geq$ on $C\left(\Omega_{X_{\beta}}\right.$, Z) by letting $f \geq g$ if $f(x) \geq g(x)$ for all $x \in \Omega_{X_{\beta}}$. We write $f>g$ if $f \geq g$ and $f \neq g$. If $x=\left(\left[x_{n}\right]_{n}\right)_{n \in \mathrm{N}_{0}} \in \Omega_{X_{\beta}}$ and $a \in \mathscr{P}_{1}\left(x_{1}\right)$, then $\left(\left[a x_{n+1}\right]_{n}\right)_{n \in \mathrm{N}_{0}}$ will belong to $\Omega_{X_{\beta}}$. We will denote this element by $a x$. We use $\lambda$ to denote the map on $C\left(\Omega_{X_{\beta}}, \mathrm{Z}\right)$ given by

$$
\lambda(f)(x)=\sum_{a \in \mathscr{P}_{1}\left(x_{1}\right)} f(a x)
$$

for all $x=\left(\left[x_{n}\right]_{n}\right)_{n \in \mathrm{N}_{0}} \in \Omega_{X_{\beta}}$ and all $f \in C\left(\Omega_{X_{\beta}}, \mathrm{Z}\right)$, cf. [4].

We may by [15], [16], and [4] compute $\left(\Delta_{\beta}, \Delta_{\beta}^{+}\right)$as the limit of the inductive system $\left(C\left(\Omega_{X_{\beta}}, \mathrm{Z}\right), \lambda\right)_{n \in \mathrm{N}_{0}}$ of abelian groups. If $\left(\kappa_{n}\right)_{n \in \mathrm{N}_{0}}$ denotes the sequence of maps $\kappa_{n}: C\left(\Omega_{X_{\beta}}, \mathrm{Z}\right) \rightarrow \Delta_{\beta}$ given according to the universal property of inductive limits, such that $\kappa_{n+1} \circ \lambda=\kappa_{n}$ for all $n \in \mathrm{N}_{0}$, then we denote by $\Delta_{\beta}^{+}$ the positive cone of $\Delta_{\beta}$ defined by

$$
\Delta_{\beta}^{+}=\left\{\kappa_{n}(f) \mid n \in \mathbf{N}_{0}, f \geq 0\right\} .
$$

The automorphism $\tau_{\beta}$ on $\Delta_{\beta}$ is then defined by requiring that $\tau_{\beta} \circ \kappa_{n}=\kappa_{n} \circ \lambda$ for all $n \in \mathrm{N}_{0}$. The element $\kappa_{0}\left(1_{\Omega_{X_{\beta}}}\right)$ is a distinguished order unit in $\left(\Delta_{\beta}, \Delta_{\beta}^{+}\right)$ corresponding to the unit of the fixed point algebra, and we denote it by $\mathbf{1}_{\Delta_{\beta}}$.

For $l \geq n$, let $W_{n}^{l}$ be the set

$$
W_{n}^{l}=\left\{\begin{array}{l|l}
u \in W_{n} & \begin{array}{l}
u_{1} u_{2} \cdots\left(u_{n-l}+1\right) \in W \\
u_{1} u_{2} \cdots\left(u_{k}+1\right) \notin W \text { for } n-l<k \leq n
\end{array}
\end{array}\right\}
$$

Of course the elements will fail to be in $W$ when $u_{n-l}$ or $u_{k}$ equals $\lceil\beta\rceil-1$. When $l=n$ the first condition is considered vacuously true. 
Lemma 2.1. For all $n, l \in \mathrm{N}_{0}$ with $l \leq n$, we have

$$
W_{n}^{l}=\left\{u \zeta_{0} \zeta_{1} \cdots \zeta_{l-1} \mid u \in W_{n-l}^{0}\right\}
$$

Proof. If $u \in W_{n-l}^{0}$, then $u_{1} u_{2} \cdots\left(u_{n-l}+1\right) \in W$ and

$$
u \zeta_{0} \zeta_{1} \cdots\left(\zeta_{k-1-(n-l)}+1\right) \notin W \quad \text { for } \quad n-l<k \leq n .
$$

Thus $\left\{u \zeta_{0} \zeta_{1} \cdots \zeta_{l-1} \mid u \in W_{n-l}^{0}\right\} \subseteq W_{n}^{l}$.

If $u \in W_{n}^{l}$, then $u_{1} u_{2} \cdots u_{n-l} \in W_{n-l}^{0}$, and $u_{n-l+1} u_{n-l+2} \cdots u_{n}=\zeta_{0} \zeta_{1} \cdots$ $\zeta_{l-1}$ because if $u_{n-l+1} u_{n-l+2} \cdots u_{k}=\zeta_{0} \zeta_{1} \cdots \zeta_{k-(n-l+1)}$ for $n-l+1 \leq$ $k<n$ and $u_{k+1}<\zeta_{k+1-(n-l+1)}$, then $u_{1} u_{2} \cdots\left(u_{k+1}+1\right) \in W$. Thus $W_{n}^{l} \subseteq$ $\left\{u \zeta_{0} \zeta_{1} \cdots \zeta_{l-1} \mid u \in W_{n-l}^{0}\right\}$.

Let for $\eta \in X_{\beta}$ and $n \in \mathrm{N}_{0}, D_{n}(\eta)$ be the set

$$
\left\{l \in\{0,1, \ldots, n\} \mid \eta \leq \sigma^{l}(\zeta)\right\} .
$$

Lemma 2.2. Let $\eta \in X_{\beta}$ and $n \in \mathrm{N}_{0}$. Then $\mathscr{P}_{n}(\eta)$ is the disjoint union of those $W_{n}^{l}$ for which $l \in D_{n}(\eta)$.

Proof. Since $\left\{W_{n}^{l}\right\}_{l=0}^{n}$ is a disjoint partition of $W_{n}$, it is enough to show that if $l \in\{0,1, \ldots, n\}$ and $u \in W_{n}^{l}$, then $u \eta \in X_{\beta}$ if and only if $\eta \leq \sigma^{l}(\zeta)$.

It follows from Lemma 2.1 that if $l \in\{0,1, \ldots, n\}, u \in W_{n}^{l}$ and $\eta \leq \sigma^{l}(\zeta)$, then $u \eta \in X_{\beta}$. If, on the other hand, $l \in\{0,1, \ldots, n\}, u \in W_{n}^{l}$ and $u \eta \in X_{\beta}$, then

$$
\zeta_{0} \zeta_{1} \cdots \zeta_{l-1} \eta=\sigma^{n-l}(u \eta) \leq \zeta
$$

and so $\eta \leq \sigma^{l}(\zeta)$.

The following notation will be useful: For $x=\left([x]_{n}\right)_{n \in \mathrm{N}_{0}} \in \Omega_{X_{\beta}}$, we define $\mathscr{P}_{n}(x)=\mathscr{P}_{n}\left(x_{n}\right)$, and for $x, y \in \Omega_{X_{\beta}}$, we write $x \sim_{n} y$ if $\mathscr{P}_{n}(x)=\mathscr{P}_{n}(y)$. If $\eta, \xi \in X_{\beta}$ and $n \in \mathrm{N}_{0}$, then we will by $[\eta, \xi]_{n}$ denote the subset

$$
\left\{x \in \Omega_{X_{\beta}} \mid \exists \varepsilon \in X_{\beta}: \eta \leq \varepsilon \leq \xi \text { and } \mathscr{P}_{n}(\varepsilon)=\mathscr{P}_{n}(x)\right\}
$$

of $\Omega_{X_{\beta}}$.

REMARK 2.3. It follows from the construction of $\Omega_{X_{\beta}}$ and its topology that $C\left(\Omega_{X_{\beta}}, \mathrm{Z}\right)$ is generated by $\left(1_{[\eta, \eta]_{n}}\right)_{\eta \in X_{\beta}, n \in \mathrm{N}_{0}}$, and it follows from Lemma 2.2 that if $\eta \in X_{\beta}, n \in \mathrm{N}_{0}$ and $k, l \in\{0,1, \ldots, n\}$ are such that $\sigma^{k}(\zeta)<\eta \leq$ $\sigma^{l}(\zeta)$ and there exists no $i \in\{0,1, \ldots, n\}$ such that $\sigma^{k}(\zeta)<\sigma^{i}(\zeta)<\sigma^{l}(\zeta)$, then

$$
[\eta, \eta]_{n}=\left[0, \sigma^{l}(\zeta)\right]_{n} \backslash\left[0, \sigma^{k}(\zeta)\right]_{n}=\left[0, \sigma^{l}(\zeta)\right]_{l} \backslash\left[0, \sigma^{k}(\zeta)\right]_{k}
$$

So $C\left(\Omega_{X_{\beta}}, \mathrm{Z}\right)$ is generated by $\left(1_{\left[0, \sigma^{n}(\zeta)\right]_{n}}\right)_{n \in \mathrm{N}_{0}}$. 
The following result is from [12]. We include a proof for the sake of selfcontainedness.

Lemma 2.4 ([12, Lemma 4.4]). Let $n, l \in \mathrm{N}_{0}$ with $l \leq n$. Then

$$
\lambda\left(1_{\left[0, \sigma^{l}(\zeta)\right]_{n}}\right)=\zeta_{l} 1_{\Omega_{X_{\beta}}}+1_{\left[0, \sigma^{l+1}(\zeta)\right]_{n+1}} .
$$

Proof. It follows from Lemma 2.2 that if $\eta, \xi \in X_{\beta}$ and $k \in \mathrm{N}_{0}$, then $\mathscr{P}_{k}(\eta)=\mathscr{P}_{k}(\xi)$ if and only if $D_{k}(\eta)=D_{k}(\xi)$, so if $a \in \mathfrak{a}$ and $x \in \Omega_{X_{\beta}}$, then

$$
\begin{aligned}
a x \in & {\left[0, \sigma^{l}(\zeta)\right]_{n} } \\
& \Longleftrightarrow \exists \eta \leq \sigma^{l}(\zeta): D_{n}(\eta)=D_{n}\left(a x_{n+1}\right) \\
& \Longleftrightarrow\left(a<\zeta_{l}\right) \vee\left(a=\zeta_{l} \wedge \exists \xi \leq \sigma^{l+1}(\zeta): D_{n+1}(\xi)=D_{n+1}\left(x_{n+1}\right)\right) \\
& \Longleftrightarrow\left(a<\zeta_{l}\right) \vee\left(a=\zeta_{l} \wedge x \in\left[0, \sigma^{l+1}(\zeta)\right]_{n+1}\right),
\end{aligned}
$$

from which the lemma follows.

We denote for every $n \in \mathrm{N}_{0}$ the function $\lambda^{n}\left(1_{\Omega_{X_{\beta}}}\right)$ by $f_{n}$.

Lemma 2.5. For every $n \in \mathrm{N}_{0}$ and every $x \in \Omega_{X_{\beta}}$, we have

$$
f_{n}(x)=\# \mathscr{P}_{n}(x) .
$$

Proof. Easily proved by induction over $n$.

From this point onwards, we need to specialize to the case in point of nonsofic $X_{\beta}$. As seen in [1, Proposition 4.2] and [12, Proposition 3.8(ii)] the sofic case is characterized by $\zeta$ being eventually periodic, and it is precisely through the condition $\sigma^{k}(\zeta) \neq \sigma^{l}(\zeta)$ for $k \neq l$ that the property enters our proof below.

Lemma 2.6. Let $n \in \mathrm{N}_{0}$.

(1) For any $x, y \in \Omega_{X_{\beta}}$, if $x \sim_{n} y$ then $f_{n}(x)=f_{n}(y)$.

(2) If $X_{\beta}$ is not sofic, then there exist $x, y \in \Omega_{X_{\beta}}$ for which $x \sim_{n} y$ and $f_{n+1}(x) \neq f_{n+1}(y)$.

Proof. (1) follows from Lemma 2.5. For (2) choose $l \in\{0,1, \ldots, n\}$ such that $\sigma^{n+1}(\zeta)<\sigma^{l}(\zeta)$ and such that there exists no $k \in\{0,1, \ldots, n\}$ such that $\sigma^{n+1}(\zeta)<\sigma^{k}(\zeta)<\sigma^{l}(\zeta)$. It then follows from Lemma 2.2 that $\mathscr{P}_{n}\left(\sigma^{n+1}(\zeta)\right)=\mathscr{P}_{n}\left(\sigma^{l}(\zeta)\right)$ and $\mathscr{P}_{n+1}\left(\sigma^{l}(\zeta)\right) \subsetneq \mathscr{P}_{n+1}\left(\sigma^{n+1}(\zeta)\right)$. So if we let

$$
x=\left(\left[x_{i}\right]_{i}\right)_{i \in \mathrm{N}_{0}} \in \Omega_{X_{\beta}}
$$

and

$$
y=\left(\left[y_{i}\right]_{i}\right)_{i \in \mathrm{N}_{0}} \in \Omega_{X_{\beta}},
$$


where $x_{i}=\sigma^{n+1}(\zeta)$ and $y_{i}=\sigma^{l}(\zeta)$ for every $i \in \mathrm{N}_{0}$, then $x \sim_{n} y$ and $f_{n+1}(x)>f_{n+1}(y)$.

Lemma 2.7. When $X_{\beta}$ is not sofic, then we have for every $n \in \mathrm{N}_{0}$ that

$$
\left\{f_{0}, f_{1}, \ldots, f_{n}\right\}
$$

is linearly independent in $C\left(\Omega_{X_{\beta}}, \mathrm{C}\right)$.

Proof. The lemma is certainly true for $n=0$. Assume that $\left\{f_{0}, f_{1}, \ldots, f_{n}\right\}$ is linearly independent and that

$$
b_{0} f_{0}+b_{1} f_{1}+\cdots+b_{n+1} f_{n+1}=0 .
$$

Choose by property (2) of Lemma 2.6 two elements $x, y \in \Omega_{X_{\beta}}$ such that $x \sim_{n} y$ and $f_{n+1}(x) \neq f_{n+1}(y)$. Then since

$$
\begin{aligned}
-b_{i+1} f_{n+1}(x)=\left(b_{0} f_{0}+\right. & \left.\cdots+b_{n} f_{n}\right)(x) \\
& =\left(b_{0} f_{0}+\cdots+b_{n} f_{n}\right)(y)=-b_{n+1} f_{n+1}(y),
\end{aligned}
$$

$b_{n+1}$ must be equal to 0 , and therefore $b_{0}=b_{1}=\cdots=b_{n}=0$ by the inductive hypothesis.

Definition 2.8. Let $\Phi: \bigoplus_{k \in \mathrm{N}_{0}} \mathrm{Z} \rightarrow C\left(\Omega_{X_{\beta}}, \mathrm{Z}\right)$ be the group morphism defined by

$$
\Phi\left(b_{0}, b_{1}, \ldots\right)=\sum_{k \in \mathrm{N}_{0}} b_{k} f_{k} .
$$

The following result establishes a group isomorphism like in [12, Corollary $4.3 \& 4.6]$, but with a set of generators better suited for an analysis of the positive cone.

Proposition 2.9. The group morphism $\Phi$ is an isomorphism when $X_{\beta}$ is not sofic.

Proof. That $\Phi$ is injective follows from Lemma 2.7. It follows from Remark 2.3 that in order to prove that $\Phi$ is surjective, it is enough to show that $1_{\left[0, \sigma^{n}(\zeta)\right]_{n}} \in \Phi\left(\bigoplus_{k \in \mathrm{N}_{0}}\right.$ Z) for every $n \in \mathbf{N}_{0}$, and since

$$
\lambda\left(\Phi\left(\bigoplus_{k \in N_{0}} \mathrm{z}\right)\right) \subseteq \Phi\left(\bigoplus_{k \in \mathrm{N}_{0}} \mathrm{z}\right),
$$

this follows from Lemma 2.4 . 


\section{Computing the dimension triple}

Let $M_{\beta}=\sum_{n \in \mathrm{N}_{0}}(n+1) \zeta_{n} \beta^{-n-1}$ (cf. [11, Corollary 3.6]) and for every $x=$ $\left(\left[x_{l}\right]_{l}\right)_{l \in \mathrm{N}_{0}} \in \Omega_{X_{\beta}}$, let $D(x)$ be the set $\left\{n \in \mathrm{N}_{0} \mid \sigma^{n}(\zeta) \geq x_{n}\right\}$. Define (cf. the function $h_{\beta}:[0,1] \rightarrow \mathrm{R}$ in [17]) a function $F$ on $\Omega_{X_{\beta}}$ by

$$
F(x)=M_{\beta}^{-1} \sum_{n \in D(x)} \beta^{-n} .
$$

LEMma 3.1. The sequence $\left(\beta^{-n} f_{n}\right)_{n \in \mathrm{N}_{0}}$ of functions converges uniformly on $\Omega_{X_{\beta}}$ to $F$.

PRoOF. It follows from Lemma 2.1, 2.2 and 2.5 that

$$
f_{n}(x)=\# \mathscr{P}_{n}(x)=\sum_{l \in D_{n}\left(x_{n}\right)} \# W_{n}^{l}=\sum_{l \in D_{n}\left(x_{n}\right)} \# W_{n-l}^{0}
$$

for all $n \in \mathrm{N}_{0}$ and $x=\left(\left[x_{k}\right]_{k}\right)_{k \in \mathrm{N}_{0}} \in \Omega_{X_{\beta}}$.

Let

$$
K=\sup _{i \in \mathrm{N}_{0}} i\left|\beta^{-i} \# W_{i}^{0}-M_{\beta}^{-1}\right| .
$$

According to the proof of [11, Corollary 3.6] we have $K<\infty$. So for all $n \in \mathrm{N}_{0}$ and $x=\left(\left[x_{k}\right]_{k}\right)_{k \in \mathrm{N}_{0}} \in \Omega_{X_{\beta}}$ we have

$$
\begin{aligned}
\mid \beta^{-n} & f_{n}(x)-F(x) \mid \\
& =\left|\sum_{l \in D_{n}\left(x_{n}\right)} \beta^{-n} \# W_{n-l}^{0}-M_{\beta}^{-1} \sum_{l \in D(x)} \beta^{-l}\right| \\
& \leq\left(\sum_{l \in D_{n-1}\left(x_{n}\right)} \beta^{-l}\left|\beta^{l-n} \# W_{n-l}^{0}-M_{\beta}^{-1}\right|\right)+\beta^{-n}+M_{\beta}^{-1} \sum_{l \geq n} \beta^{-l} \\
& \leq\left(\sum_{l \in D_{n-1}\left(x_{n}\right)} \beta^{-l} K /(n-l)\right)+\beta^{-n}+M_{\beta}^{-1} \beta^{1-n} /(\beta-1) \\
& \leq K\left(\sum_{i=1}^{n} \beta^{i-n} / i\right)+\beta^{-n}+M_{\beta}^{-1} \beta^{1-n} /(\beta-1) \rightarrow 0
\end{aligned}
$$

as $n \rightarrow \infty$. To see that the first term converges one may for instance for each $k$ majorize the first $n-\lceil n / k\rceil$ terms by $\beta^{i-n}$ and the last $\lceil n / k\rceil$ terms by $1 / n$.

It follows from Proposition 2.9 that the map $\psi$

$$
\sum_{k \in \mathrm{N}_{0}} b_{k} f_{k} \mapsto \sum_{k \in \mathrm{N}_{0}} b_{k} \beta^{k}
$$


is a well-defined linear functional from $C\left(\Omega_{X_{\beta}}, \mathrm{Z}\right)$ to $\mathrm{R}$ when $X_{\beta}$ is not sofic.

In the proof below, we use the notation $T_{\beta}(1)=\lim _{x \nearrow 1} T_{\beta}(x)$.

LEMma 3.2. The functional $\psi$ has the following two properties:

(1) If $f, g \in C\left(\Omega_{X_{\beta}}, \mathrm{Z}\right)$ and $f>g$, then $\psi(f)>\psi(g)$,

(2) if $f \in C\left(\Omega_{X_{\beta}}, \mathrm{Z}\right)$, then $\left(\beta^{-n} \lambda^{n}(f)\right)_{n \in \mathrm{N}_{0}}$ converges uniformly on $\Omega_{X_{\beta}}$ to $\psi(f) F$.

Proof. That property (2) holds follows from Lemma 3.1.

Let $f, g \in C\left(\Omega_{X_{\beta}}, \mathbf{Z}\right)$ and $f>g$. It then follows from Remark 2.3 that $f-g$ can be written as a linear combination of elements of the form $1_{\left[0, \sigma^{l}(\zeta)\right]_{l}}-$ $1_{\left[0, \sigma^{k}(\zeta)\right]_{k}}$ with $\sigma^{l}(\zeta)>\sigma^{k}(\zeta)$ and with all the coefficients positive. It follows from Lemma 2.4 that $\psi\left(1_{\left[0, \sigma^{l}(\zeta)\right]_{l}}\right)=T_{\beta}^{l}(1)$ for all $l \in \mathbf{N}_{0}$, and since (cf. [11, Proposition 3.2]) $\sigma^{l}(\zeta)>\sigma^{k}(\zeta)$ implies $T_{\beta}^{l}(1)>T_{\beta}^{k}(1)$, we get that $\psi(f-g)>0$ and thus $\psi(f)>\psi(g)$.

Theorem 3.3. When $X_{\beta}$ is not sofic, the dimension triple $\left(\Delta_{\beta}, \Delta_{\beta}^{+}, \tau_{\beta}\right)$ is isomorphic to $\left(\mathrm{Z}\left[t, t^{-1}\right], P C_{\beta}, \mu\right)$, where

$$
P C_{\beta}=\left\{p \in \mathrm{Z}\left[t, t^{-1}\right] \mid p=0 \text { or } p(\beta)>0\right\},
$$

and $\mu: \mathrm{Z}\left[t, t^{-1}\right] \rightarrow \mathrm{Z}\left[t, t^{-1}\right]$ is multiplication by $t$. Under this isomorphism, the distinguished order unit $\mathbf{1}_{\Delta_{\beta}}$ is mapped to 1 .

PRoof. For every $i \in \mathrm{N}_{0}$, let $\iota_{i}$ be the group morphism from $C\left(\Omega_{X_{\beta}}, \mathrm{Z}\right)$ to $\mathrm{Z}\left[t, t^{-1}\right]$ defined by

$$
\iota_{i}\left(\sum_{k \in \mathrm{N}_{0}} b_{k} f_{k}\right)=\sum_{k \in \mathrm{N}_{0}} b_{k} t^{k-i} .
$$

It follows from Proposition 2.9 that $\iota_{i}$ is well-defined and injective. Because $\iota_{i+1} \circ \lambda=\iota_{i}$ for every $i \in \mathrm{N}_{0}$, the $\iota_{i}$ 's induce a group morphism $\iota$ from $\Delta_{\beta}$ to $Z\left[t, t^{-1}\right]$. Since $\iota_{i}$ is injective for every $i \in \mathrm{N}_{0}, \iota$ is injective. Further, the following equality holds:

$$
\bigcup_{i \in \mathrm{N}_{0}} \iota_{i}\left(C\left(\Omega_{X_{\beta}}, \mathrm{Z}\right)\right)=\mathrm{Z}\left[t, t^{-1}\right] .
$$

Consequently, $\iota$ is also surjective and thus an isomorphism, and since $\iota_{i} \circ \lambda=$ $\mu \circ \iota_{i}$ for every $i \in \mathrm{N}_{0}$, we have that $\iota \circ \tau_{\beta}=\mu \circ \iota$.

Let $\sum_{k=-l}^{m} b_{k} t^{k} \in \mathrm{Z}\left[t, t^{-1}\right]$ with $\sum_{k=-l}^{m} b_{k} \beta^{k}>0$, and let $f=\sum_{k=-l}^{m} b_{k} f_{k+l}$. We then have that

$$
\psi(f)=\beta^{l} \sum_{k=-l}^{m} b_{k} \beta^{k}>0,
$$


and since it follows from Lemma 3.2 that $\left(\beta^{-n} \lambda^{n}(f)\right)_{n \in \mathrm{N}_{0}}$ converges uniformly on $\Omega_{X_{\beta}}$ to $\psi(f) F$, and $F(x) \geq M_{\beta}^{-1}$ (since $0 \in D(x)$ for every $x \in \Omega_{X_{\beta}}$ ), there exists an $n \in \mathrm{N}_{0}$ such that $\sum_{k=-l}^{m} b_{k} f_{k+l+n}$ is a strictly positive function, and hence

$$
\sum_{k=-l}^{m} b_{k} t^{k}=\iota_{l+n}\left(\sum_{k=-l}^{m} b_{k} f_{k+l+n}\right) \in \iota\left(\Delta_{\beta}^{+}\right) .
$$

Let us denote the linear functional $p \mapsto p(\beta)$ from $\mathrm{Z}\left[t, t^{-1}\right]$ to $\mathrm{R}$ by $\Psi$. If $f \in C\left(\Omega_{X_{\beta}}, \mathrm{Z}\right)$ and $f>0$, then $\psi(f)>0$ according to Lemma 3.2, and since $\beta^{n} \Psi \circ \iota_{n}=\psi$ for every $n \in \mathrm{N}_{0}$, we have that $\Psi\left(\iota_{n}(f)\right)>0$ for all $n \in \mathrm{N}_{0}$, which shows that $\iota\left(\Delta_{\beta}^{+}\right) \subseteq P C_{\beta}$.

Finally, we note that $\iota\left(\mathbf{1}_{\Delta_{\beta}}\right)=\iota_{0}\left(f_{0}\right)=1$.

COROLlary 3.4. The dimension groups associated in [12] and in [21], respectively, to any $\beta$-shift, coincide.

Proof. The sofic case was solved in [21, Proposition 10.6], and the nonsofic case is got by comparing the previous theorem to [21, Proposition 10.5].

Corollary 3.5. The AF algebras $\mathscr{F}_{\beta}^{\infty}$ and $F_{T_{\beta}}$ defined in [12] and in [7], respectively, are isomorphic for any $\beta$.

Proof. The sofic case was solved in [7, Corollary 15.3], and the non-sofic case is got by comparing the dimension group in the previous theorem, along with the distinguished order unit, to that of [21, Proposition 10.6].

\section{REFERENCES}

1. Blanchard, F., $\beta$-expansions and symbolic dynamics, Theoret. Comput. Sci. 65 (1989), 131 141.

2. Bratteli, O., Inductive limits of finite dimensional $C^{*}$-algebras, Trans. Amer. Math. Soc. 171 (1972), 195-234.

3. Carlsen, T. M., Cuntz-Pimsner $C^{*}$-algebras associated with subshifts, to appear in Internat. J. Math.

4. Carlsen, T. M., and Eilers, S., Matsumoto $K$-groups associated to certain shift spaces, Doc. Math. 9 (2004), 639-671 (electronic).

5. Carlsen, T. M., and Matsumoto, K., Some remarks on the $C^{*}$-algebras associated with subshifts, Math. Scand. 90 (2004), 145-160.

6. Carlsen, T. M., and Silvestrov, S., $C^{*}$-crossed products and shift spaces, submitted for publication, arXiv:math.OA/0512488, 2005.

7. Deaconu, V., and Shultz, F., $C^{*}$-algebras associated with interval maps, Trans. Amer. Math. Soc. 359 (2007), 1889-1924.

8. Effros, E. G., Handelman, D. E., and Shen, C. L., Dimension groups and their affine representations, Amer. J. Math. 102 (1980), 385-407.

9. Elliott, G. A., On the classification of inductive limits of sequences of semisimple finitedimensional algebras, J. Algebra 38 (1976), 29-44. 
10. Goodearl, K. R., Partially Ordered Abelian Groups with Interpolation, American Mathematical Society, Providence, R.I., 1986.

11. Ito, S., and Takahashi, Y., Markov subshifts and realization of $\beta$-expansions, J. Math. Soc. Japan 26 (1974), 33-55.

12. Katayama, Y., Matsumoto, K., and Watatani, Y., Simple $C^{*}$-algebras arising from $\beta$-expansion of real numbers, Ergodic Theory Dynam. Systems 18 (1998), 937-962.

13. Lind, D., and Marcus, B., An Introduction to Symbolic Dynamics and Coding, Cambridge University Press, Cambridge, 1995.

14. Matsumoto, K., On $C^{*}$-algebras associated with subshifts, Internat. J. Math. 8 (1997), 357374.

15. Matsumoto, K., Dimension groups for subshifts and simplicity of the associated $C^{*}$-algebras, J. Math. Soc. Japan 51 (1999), 679-698.

16. Matsumoto, K., Presentations of subshifts and their topological conjugacy invariants, Doc. Math. 4 (1999), 285-340 (electronic).

17. Parry, W., On the $\beta$-expansions of real numbers, Acta Math. Acad. Sci. Hungar. 11 (1960), 401-416.

18. Rényi, A., Repesentations for real numbers and their ergodic properties, Acta Math. Acad. Sci. Hungar. 8 (1957), 477-493.

19. Schmeling, J., Symbolic dynamics for $\beta$-shifts and self-normal numbers, Ergodic Theory Dynam. Systems 17 (1997), 675-694.

20. Shultz, F., Dimension groups for interval maps, New York J. Math. 11 (2005), 477-517 (electronic).

21. Shultz, F., Dimension groups for interval maps II: The transitive case, arXiv:math.DS/0405467, to appear in Ergodic Theory Dynam. Systems.

SCHOOL OF MATHEMATICS AND STATISTICS

UNIVERSITY OF NEW SOUTH WALES

SYDNEY, NSW, 2052

AUSTRALIA

E-mail: teresa@unsw.edu.au

INSTITUT FOR MATEMATISKE FAG

KØBENHAVNS UNIVERSITET

UNIVERSITETSPARKEN 5

2100 COPENHAGEN Ø

DENMARK

E-mail: eilers@math.ku.dk
MATHEMATICHES INSTITUT

WESTFÄLISCHE WILHELMS-UNIVERSITÄT MÜNSTER

EINSTEINSTRAßE 62

48149 MÜNSTER

GERMANY

E-mail: toke@math.uni-muenster.de

PRESENT ADDRESS:

SCHOOL OF MATHEMATICAL AND PHYSICAL SCIENCES

UNIVERSITY OF NEWCASTLE

CALLAGHAN, NSW 2308

AUSTRALIA

E-mail: Toke.Carlsen@newcastle.edu.au 